\title{
A Method to Evaluate the Temperature Profile in a Superconducting Magnet During a Quench
}

\author{
V. Maroussov, A. Siemko \\ CERN, Geneva, Switzerland
}

\begin{abstract}
A simple method to derive the temperature profile in a superconducting magnet during a quench from measured voltage signals is described. The method was applied to several Large Hadron Collider single aperture dipole models. These measurements show the strong correlation between parameters of the magnet protection system and powering on the one hand and the resulting temperature gradient in the magnet coil on the other. The method allows the localisation of critical points in the magnet design, in particular, the efficiency of the magnet protection system can be evaluated.
\end{abstract}

\section{INTRODUCTION}

Large-scale superconducting magnets, such as the Large Hadron Collider (LHC) main ring dipoles $[1,2]$ should be designed to quench safely in various conditions of accelerator operation. For such magnets the high density of stored electromagnetic energy [1] implies an efficient protection system in case of a quench. As the basic process of a quench is the conversion of stored electromagnetic energy into heat the primary issue of magnet protection is to keep the temperature rise within proper limits in order to eliminate the danger of local overheating and resulting materials damage.

The protection of each individual LHC main dipole is based on the so-called quench heater strips. Their role is to spread the transition from superconducting to normal state over as large volume as possible in order to achieve more uniform energy dissipation and to limit the highest hot spot temperature.

Even if the temperature rise is kept within bounds large temperature gradients up to a few hundreds of degrees can develop across the magnet coil parts. It was observed that large temperature gradients [3] can influence significantly the quench performance of the magnet and can even cause performance degradation.

In this paper a simple method to derive the temperature profile from measured voltage signals in a superconducting magnet during a quench is described. The method allows the evaluation of the dynamics of temperature development in different parts of superconducting coil and the localisation of critical points in the magnet design at various powering conditions and, in particular, assessment of the magnet protection system.

\section{GENERAL DESCRIPTION OF THE METHOD}

\section{A. Basic Equations}

For a non-linear purely inductive circuit the differential inductance $L(I)$ can be defined as the ratio of the infinitesimal changes of current $I$ and flux $\Phi$ through the winding. In such a case the energy equation which couples the infinitesimal changes of stored energy $E$ and square of current remains rather similar to that for a linear circuit:

$$
L(I)=\frac{d \Phi}{d I} \quad \Rightarrow \quad d E=\frac{L(I)}{2} d\left(I^{2}\right)
$$

For the integral equations this similarity disappears. We can, however, define a function $L_{\Phi}(I)$ such that $\Phi=L_{\Phi}(I) \cdot I$ and a function $L_{E}(I)$ such that $E=1 / 2 \cdot L_{E}(I) I^{2}$, but these functions in general are different:

$$
\begin{aligned}
& L_{\Phi}(I)=\frac{1}{I} \int_{0}^{I} L\left(I^{\prime}\right) d I^{\prime} \\
& L_{E}(I)=\frac{2}{I^{2}} \int_{0}^{I} L\left(I^{\prime}\right) I^{\prime} d I^{\prime}
\end{aligned}
$$

The magnet equivalent circuit during a quench can be drawn as a serial connection of a non-linear inductance $L(I)$ and a time dependent resistance $R(t)$. In general, $R(t)$ changes in a different way from quench to quench. The energy equation which links the current $I$ through the magnet, the voltage $V$ on the magnet and Joule heat released in the magnet during a quench can be written as follows:

$$
\begin{aligned}
& Q(t)=\int_{t_{o}}^{t}\left(V\left(t^{\prime}\right)-V_{\text {inductive }}\right) I\left(t^{\prime}\right) d t^{\prime}= \\
& \int_{t_{o}}^{t}\left(V\left(t^{\prime}\right)-L(I) \frac{d I}{d t}\right) I\left(t^{\prime}\right) d t^{\prime}=\ldots \\
& =\int_{t_{o}}^{t} V\left(t^{\prime}\right) I\left(t^{\prime}\right) d t^{\prime}+\frac{1}{2}\left(L_{E}\left[I\left(t_{0}\right)\right] I\left(t_{0}\right)^{2}-L_{E}[I(t)] I(t)^{2}\right)
\end{aligned}
$$

Here it is assumed that the time $t_{0}$ is chosen before the quench, when the magnet coil is in the superconducting state. 
Let us consider the case when $V$ is not the total voltage on the magnet, but the voltage is measured on one turn of the magnet coil. The inductive voltage across a single turn is proportional to the time derivative of the magnetic flux through the turn. Assuming that during a quench the ratio of the flux through the turn to the whole flux through the magnet coil remains constant (in general, it can change with the iron yoke saturation - see chapter below) the ratio of inductive voltage $V_{\text {inductive }}$ at any turn to the whole inductive voltage on the magnet remains constant, although the magnet inductance may be not constant. At this condition the equation which is similar to (3) is applicable for a single turn of the magnet coil :

$$
\begin{aligned}
& Q(t)=\int_{t_{o}}^{t} V\left(t^{\prime}\right) I\left(t^{\prime}\right) d t^{\prime} \\
& +\frac{L_{n}}{L_{E}(0)} \frac{1}{2}\left(L_{E}\left[I\left(t_{0}\right)\right] I\left(t_{0}\right)^{2}-L_{E}[I(t)] I(t)^{2}\right) \\
& \text { here } L_{n}=\frac{V_{\text {inductive }}^{(\text {turn })}}{V_{\text {inductive }}^{(\text {magnet }}} L_{E}(0)=\frac{\Phi_{\text {turn }}}{\Phi_{\text {totul }}} L_{E}(0)
\end{aligned}
$$

Let us call $L_{n}$ "partial inductance". If the partial inductance of the turn is known, the Joule heat, which is released in the turn, can be calculated. Then, if the masses of the copper and the superconductor, and the dependencies of the specific heat versus temperature are known, the temperature of the coil turn can be calculated.

Finally, let us note that with the constancy of the flux distribution in the coil region equation (4) can be used for any part of winding, which can consist of non-integer number of turns.

\section{B. Dependence of the Flux Distribution and Inductance on the Excitation Current}

As already mentioned the way in which the inductive voltage distribution changes during the current decay is an important condition to apply the method. Therefore a numerical 2D-simulation was carried out by the MERMAID finite-element program code [3]. Two cases were considered. First, the magnetic flux distribution at the accelerator injection current of $800 \mathrm{~A}$. Second, the distribution at the ultimate

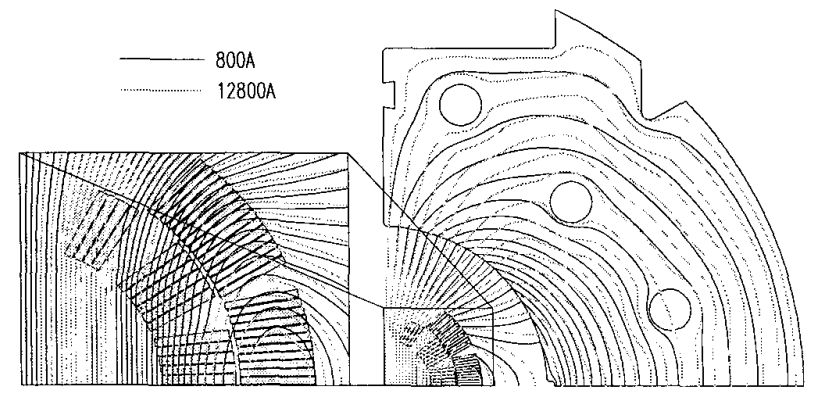

Fig. 1 Magnetic flux at $800 \mathrm{~A}$ and $12800 \mathrm{~A}$. The flux line spacing is equal to $5 \%$ and $2.5 \%$ of the full flux for the right and left picture, respectively.

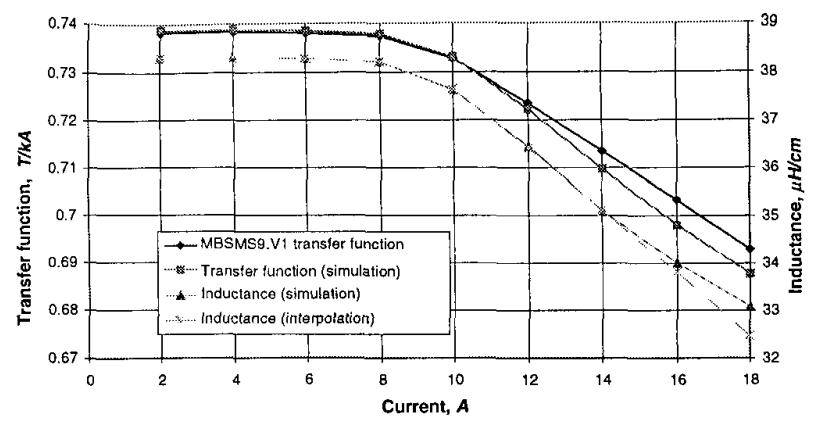

Fig. 2 Dependence of the calculated inductance $\left.L_{M}=L_{E} / 1 \mathrm{~cm}\right]$ on excitation current. Extrapolated (above $12400 \mathrm{~A}$ ) transfer function from measurements is compared to the transfer function from simulation.

current of $12800 \mathrm{~A}$. The results show that the maximum change of the ratio between magnetic flux through a single turn to the flux through the whole magnet coil does not exceed $1 \%$ for any turn. Fig. 1 presents the result graphically. As it can be seen, in the coil region (expanded view) there is no essential distortion of the magnetic flux due to the iron yoke saturation, although the flux lines picture differs very much in the yoke itself. The dependence of the integral inductance $\left.L_{M}=L_{E} / 1 \mathrm{~cm}\right]$ (per unit length of the magnet) on the excitation current was also obtained from the simulation. Results are presented in Fig.2. In order to verify the results of this simulation the calculated transfer function was compared to the real one obtained from the magnetic measurements. The difference between the curves does not exceed $0.3 \%$ for the current values below $13 \mathrm{kA}$. The difference at higher currents can be attributed to end-effects and to model differences in the iron permeability versus magnetic field.

\section{EXPERIMENTAL}

\section{A. Voltage Taps Layout in the Magnet Coil}

A typical voltage taps disposition in the LHC short $(1 \mathrm{~m}$ long) dipole model magnet is shown in Fig.3. Obviously the voltage tap cannot be placed in any arbitrary point of the magnet winding but only in a certain points which are accessible during and after the coil assembly. The placement of the voltage taps shown in Fig. 3 was chosen on the base of statistics with quench history of various magnets. In most cases the quench origin is located in the first turns of the coil pole blocks where the highest temperature and the temperature gradient can be expected.

\section{B. Inductive Voltage Distribution on the Magnet}

For a long magnet the inductive voltage distribution could be obtained with high precision from 2D computer simulations. For the LHC short dipole models used in this study it is not the case because they are not long enough with respect to the transversal coil size which is $56 \mathrm{~mm}$ and $118.6 \mathrm{~mm}$ for the inner and the outer coil diameter, respectively. Moreover some voltage tap pairs do not include 


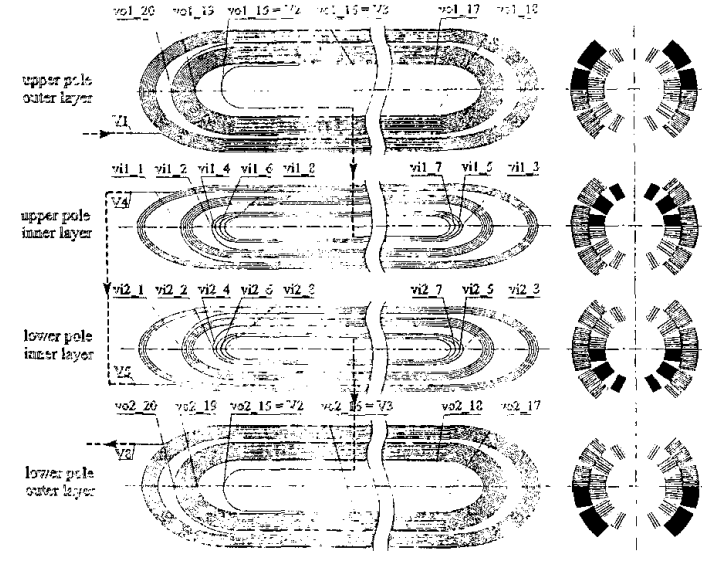

Fig. 3 Typical layout of voltage taps for the LHC short dipole model.

an integer number of turns between them. That makes the task of calculation the partial inductance 3-dimensional. Also the wiring of the voltage tap pairs becomes the dominating part of the inductive loop and the measured inductive voltages depend strongly on the geometry of this wiring. Due to circumstances mentioned above the inductive voltage distribution was not calculated but obtained directly from the quench recordings.

The idea of such measurement is rather simple. Any quench first appears at a certain single point of the magnet while most of the coil remains in superconducting state. So at the quench beginning a pure inductive voltage is measured by most of voltage taps.

For the heater-provoked quenches the quench location can be chosen because it is set by the heater location. This type of quench was used to evaluate the distribution of inductive voltages. The procedure to calculate the inductive voltage distribution in the magnet was as follows:

1) For a set of heater-provoked quenches with the initial current between 1500 and $6000 \mathrm{~A}$ the power integrals $Q_{n}=\int V_{n} I d t$ were calculated for each measured voltage $V_{n}$.

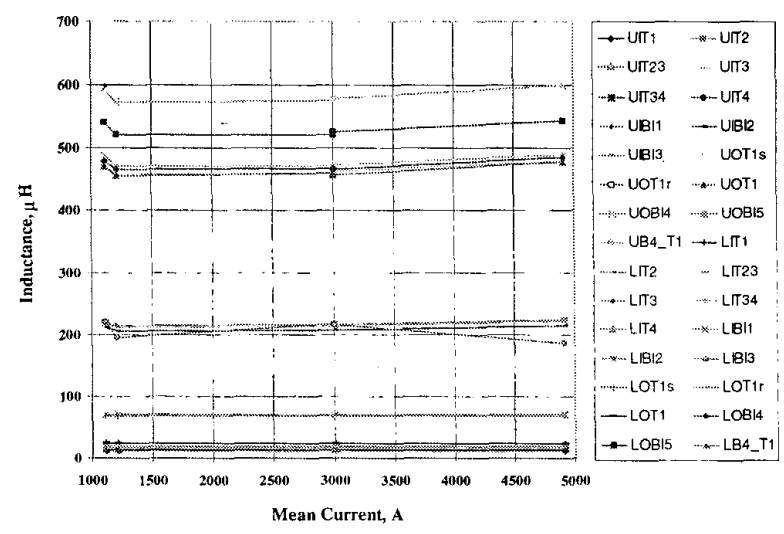

Fig. 4 Dependence of the calculated "partial inductance" $L_{n}$ on mean current during of the integration interval.
In order to minimize the $50 \mathrm{~Hz}$ noise coming from mains, the integration time interval was chosen as $[8 \mathrm{~ms}-48 \mathrm{~ms}]$ after the start of the quench.

2) From each power integral the coefficient $L_{n}$ was calculated acording to:

$$
L_{n}=\frac{2 Q_{n} L_{M}(0)}{L_{M}\left(I_{1}\right) I_{1}^{2}-L_{M}\left(I_{0}\right) I_{0}^{2}} ;
$$

here $I_{0}=I(\mathrm{t}=8 \mathrm{~ms}), I_{1}=I(\mathrm{t}=48 \mathrm{~ms})$

3) The maximum value of $L_{n}$ for every recorded voltage $V_{n}$ was found. These $L_{n}$ 's have the same physical meaning as $L_{n}$ in (4).

The procedure above can cause a mistake at the very last step (the choice of maximal value) which could have come, for instance, from a recording hardware fault. Therefore the results were verified using two simple criteria :

a) values for geometrically symmetric channels (lower pole - upper pole) should be close to each other;

b) the maximum of the set should be within 2 standard deviations from the mean value.

Results of the $L_{n}$ calculation by (5) are presented in Fig.4. As it can be seen, for most of recording channels the value of $L_{n}$ almost doesn't depend on the current. A slight variation appeared for the group of channels which recorded voltages from relatively short parts of the magnet winding. This could be expected since for a short winding section there is a high probability that during the power integration time interval [ $8 \mathrm{~ms}-48 \mathrm{~ms}$ ] the propagating quench will "capture" a relatively large part of the section. Moreover in these measuring channels, the absolute value of the signal was lower and the relative noise was higher. For the first application of the method, the recordings of the heater provoked quenches were used to measure the inductance. In order to measure the inductance in more straightforward way a fast de-excitation forced by the energy extraction system without firing the quench heaters was performed. Results of both methods were consistent.

It is worth mentioning that even for the natural quenches the quench location is usually more or less random and it is possible to choose a recording in which at the beginning of the quench part of the coil between certain voltage taps remains in a superconducting state.

\section{RESULTS}

\section{A. Dynamics of Temperature Development}

An example of the calculation of the dynamics of temperature development in different parts of the magnet coil recorded during a quench with $85 \%$ of the stored energy extracted into an external dump resistor is presented in Fig. 5. As it can be seen for this type of quench the maximum temperature in most parts of the coil appeared after about $250 \mathrm{~ms}$ from the quench beginning. The temperature of the 


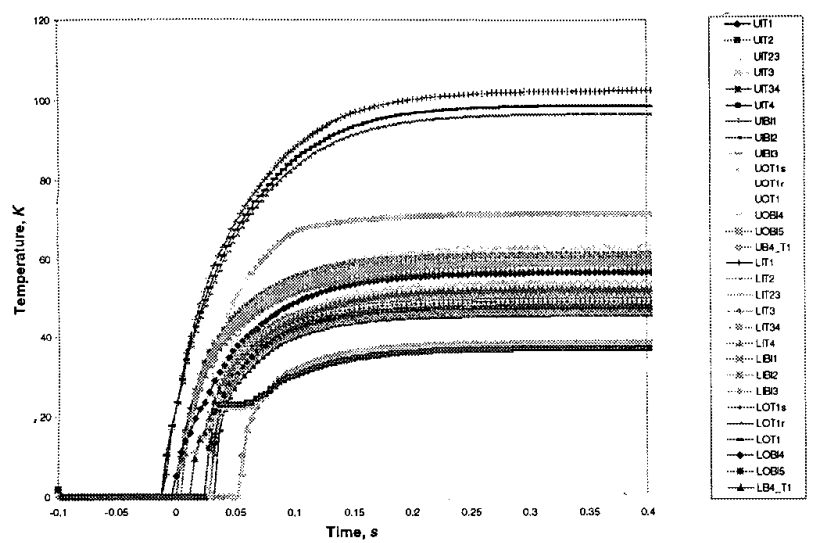

Fig. 5 The dynamics of temperature development in different parts of the magnet coil during a quench without energy extraction.

coil varies from 35 to $105 \mathrm{~K}$. Analysing a sequence of rising curves one can easily reconstruct the quench propagation in the whole coil. It is as well possible to attribute certain temperature rises to an action of the quench heaters or to the so-called quench back effect. In Fig. $6 \mathrm{a}$ and Fig. 7a two characteristic temperature plots obtained for quenches performed with (Fig. 6a cf. Fig. 5) and without (Fig. 7a) energy extraction are presented. Certainly the maximum temperature is much higher for the latter case and varies from 40 to $280 \mathrm{~K}$. As it was presented in [3] the so-called detraining effect seems to be strictly related to the hot spot temperature and temperature gradients. In certain cases this effect can even limit the performance of the magnet. Fig. 7 presents plots for two similar quenches performed without energy extraction originating in the coil inner layer (Fig. 7a) and in the coil outer layer (Fig. 7b). In spite of the quench origin in both cases the highest temperature was reached in the innermost coil turn of the outer layer. As it is shown in Fig. $6 \mathrm{~b}$ much higher temperatures and larger temperature gradients for quenches without energy extraction were recorded for the most recent LHC dipole design featuring '6block' coil design [5].
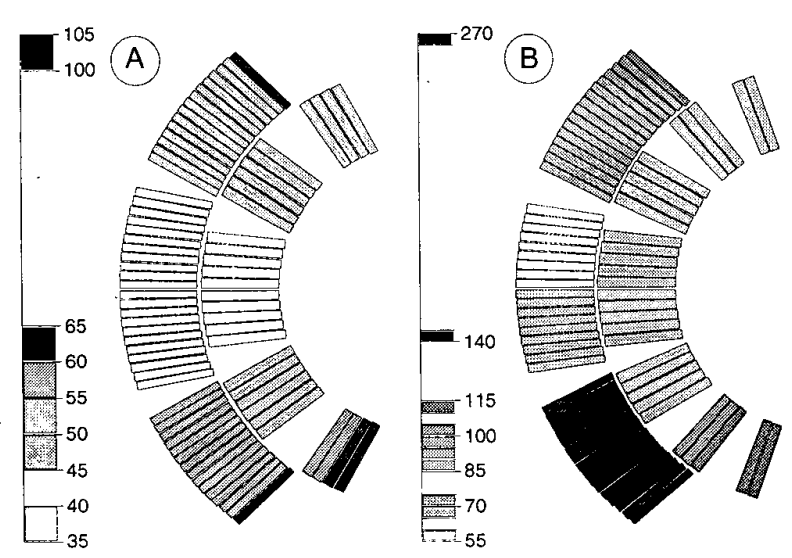

Fig. 6 Temperature profile in the coil for the quench at a) $12800 \mathrm{~A}$ with energy extraction, b) 12888 A without energy extraction (new coil design).

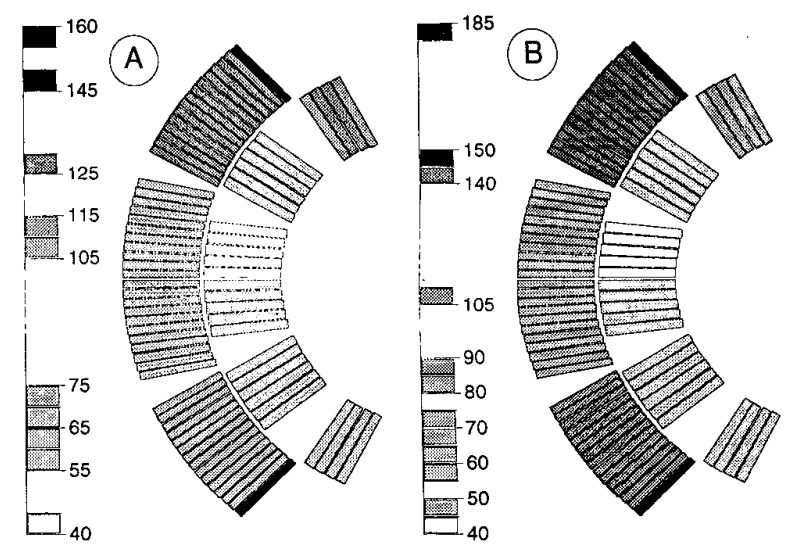

Fig. 7 Temperature profile in the coil for two quenches without energy extracted originating in (a) inner layer, (b) outer layer.

\section{CONCLUSIONS}

The proposed method allows the evaluation of temperature profiles in a superconducting magnet using usual voltage taps signals obtained during a quench. Only the transversal temperature maps were obtained so far because of the present voltage taps layout for the LHC model magnets. For magnets equipped with more detailed sets of voltage taps it is possible to obtain the longitudinal temperature profile as well.

The direct inductance measurement is a substantial part of the method and makes it self-consistent. The software presently used for the temperature calculations works off-line but the simplicity of the method allows to realize in future a version which is integrated into the quench recording software, increasing substantially the output of the analysis of routine tests.

\section{ACKNOWLEDGMENT}

The authors thank Dr. R. Bartolini for the fruitful discussions and would like to acknowledge the CERN teams involved in the construction and testing of the LHC short dipole models.

\section{REFERENCES}

[1] The LHC Study Group, "The Large Hadron Collider Conceptual Design", CERN, CERN/AC/95-05(LHC), 20 October 1995.

[2] J. Billan et al., "Test results on the long models and full scale prototype of the second generation LHC arc dipoles", this proceedings.

[3] A. Siemko et al., "Power test results of the first LHC second generation superconducting single aperture $1 \mathrm{~m}$ lang dipole models", in ICEC 16/ICMC Proceedings, Kitakyushu, Japan May 1996, T. Haruyama, T. Mitsui, K Yamafuji, Eds. Elsevier Science, 1997, pp.837-842.

[4] SIM Limited, Mermaid, Magnet design in two and three dimensions, Novosibirsk, (1994).

[5] N. Andreev et al., "State of the short dipole model program for the LHC", in EPAC'98 Proceedings, Stockholm, Sweden June 1998, pp. 311-313. 